\title{
Life-Time Analysis of Modules for Reuse in Inverse Manufacturing*
}

\author{
Yong $\mathrm{JI}^{* *}$, Hirohisa NARITA***, Lian-yi $\mathrm{CHEN}^{* * * *}$ and Hideo FUJIMOTO ${ }^{* * * *}$
}

As environmental issues have become serious, the inverse manufacturing concept is discussed to establish a sustainable society. To realize the inverse manufacturing system the prime problem is how to improve the rate of reusing the product modules. Therefore analyzing the life-time of the disassembled modules is very important before reassembling process. In this paper, a cumulative damage model is proposed to discuss the quality of the product that is assembled by reuse modules. It is supposed that modules suffer damages due to shocks and fails when the cumulative damage level exceeds the failure level. Then maintenance cost will be analyzed in order to minimize the expected maintenance cost-rate by optimal maintenance time $T$ and optimal number of damages $N$ in this cumulative damage model. The probable safe life-time of module reuse will be explored. Finally numerical examples are given to confirm the validity of the proposed model.

Key Words: Inverse Manufacturing, Reuse, Lifecycle, Life-Time, Module, Damage Model

\section{Introduction}

In past decade, environmental issues have been emphasized, and mass production and mass consumption have revealed the essential limitation of the earth. To attack these problems, a circulation type society is advocated to decrease the environmental burden. Therefore, inverse manufacturing concept is discussed to realize it, particularly in circulation production. Waste electrical and electronic equipment (WEEE) directive is promulgated (03.2003) in Europe, with the aims to reduce the waste arising from electrical and electronic equipment, to encourage reuse, recycling and recovery and to improve the environmental performance of all operators involved in the lifecycle. Eco-efficient has been evaluated by implementation of WEEE in recycling system and by achieving economy of scale in recycling system ${ }^{(1)}$. The most important

* Received 18th January, 2006 (No. 05-4284)

** Department of Systems Engineering Graduate School of Engineering, Nagoya Institute of Technology, Gokiso-cho, Showa-ku, Nagoya, Aichi 466-8555, Japan. E-mail: jiyong@vier.mech.nitech.ac.jp

*** Tsukuri College, Graduate School of Engineering, Nagoya Institute of Technology, Gokiso-cho, Showa-ku, Nagoya, Aichi 466-8555, Japan

**** Omohi College, Graduate School of Engineering, Nagoya Institute of Technology, Gokiso-cho, Showa-ku, Nagoya, Aichi 466-8555, Japan problem for realizing the circulation production is how to improve the reuse rate of the product module. Hence, the quality of the reuse module is a decisive factor. Therefore, for the operator of recycling a previous evaluation method is demanded to analyze the life-time of the reuse module for minimizing the maintenance cost when the modules are disassembled for reuse. Although many authors have proposed various concepts about inverse manufacturing, for the recycling operator there are a lot of problems that need to be solved to realize circulation production as show in Fig. 1. In general, three essential directions in inverse manufacturing system.

(1) Product design and production process. Inverse

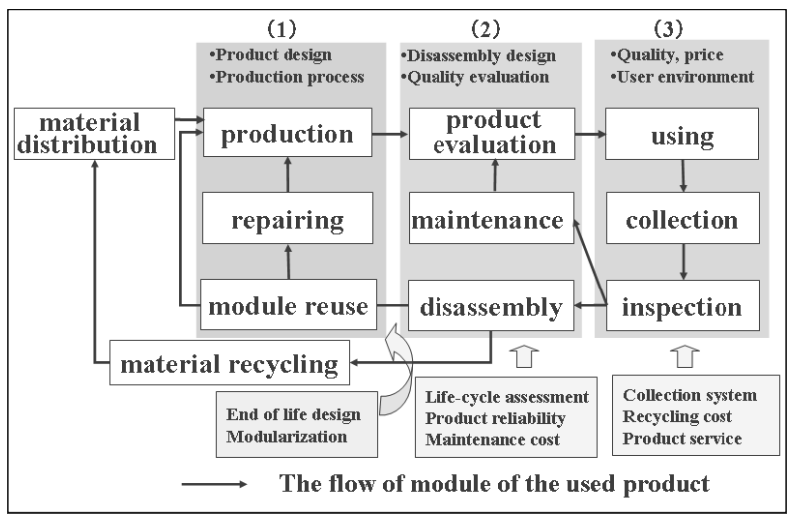

Fig. 1 Methods analysis of module reuse in each stage in product recycling 
manufacturing needs a design and evaluation of a circulation type production system $^{(2)}$. It means that design method for product structure should improve the module reuse rate and make it easy to disassemble by product life cycle design analysis ${ }^{(2)-(5)}$, and an environmental harmony type product is the purpose of the life cycle de$\operatorname{sign}^{(6)-(9)}$. In circulation process management, promoting efficiency of recycling system is necessary from remanufacturing process to reuse module supply ${ }^{(10)-(12)}$.

(2) Life cycle maintenance. There are three steps in the second direction. First, rational maintenance method decision technique should be established and computerassisted should be prepared ${ }^{(13)}$. The second is maintenance information management system for collection and practical use of a malfunction example $\mathrm{e}^{(14)-(16)}$. The third is deterioration/failure prediction technique. Parts deterioration and life of module should be analyzed before remanufacturing process.

(3) User buying behavior for remanufactured product. Product quality, product price and the user environment affect the user decision making in product life cycle. It is very important to set up product strategies for target user group base on actual quality, and production cost evaluation. Environment policy should be enacted to form user environment ${ }^{(16),(17)}$.

In fact, reliability engineering has been applied to discuss the module life-time about life cycle. The probability model is used to analyze the modules life-time on robot, and a method is proposed with Weibull distribution to discuss the failure time of modules about rapid life cycle product ${ }^{(18)-(20)}$. Though the life-time of module is discussed in these studies, the studies that only analyzed operating life did not predict the possible time for safety use, and did not analyze the cost about maintenance or repairing while discussing the life-time of the reuse modules. With the above situation as background, the objective of this study is to propose a method for predicting the optimal maintenance time of the reuse modules that minimize the maintenance cost by using a cumulative damage model and to infer the probable safe use time of modules reuse $^{(20)}$. Finally a numerical example is given for some exponential cases to confirm the validity of the model.

\section{Quality Analysis of Module Reuse and Model De- scription}

\subsection{Quality analysis of module reuse}

There are lots of problems that we have to resolve to realize the inverse manufacturing system, and for user the elements such as quality and price will be considered to decide the buying behaviors. In design process, product design makes the product easy to disassemble and recycle, production process design makes the remanufacturing more efficient. About the remanufacturing product particularly, the maintenance cost decides the economic effect

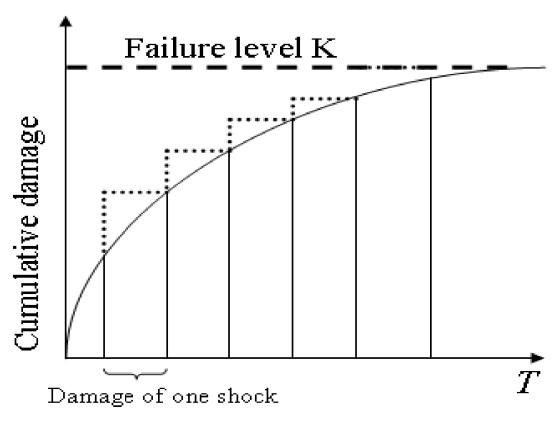

Fig. 2 Damage of shocks cause the module failure

of life cycle production, and influences the user buying behaviors. A used product is disassembled and the modules will be sent to reuse or shred as material recycle. If a product that assembled by reuse modules or reuse modules and new modules fails frequently, the user have to pay much for preparing it, and it influence circulation production. Therefore, a method is necessary to evaluate the quality of the reuse modules to guarantee the product before the reuse them. The method predicts the life of reuse and evaluates whether the module should be reused or prepared or shredded according the prediction. A damage model is applied to solve the quality problem. In using reassembled product, the cumulative damage causes the product failure when the damage exceeds to a certain level, and the failure occurs as Markov process. According to the information of used time and history records of failure, the cumulative damage model will be discussed to calculate remaining life-time of reuse modules and evaluate the probable lifetime of modules reuse. Shocks occur at random times and each shock causes a random amount of damage to module. These damages accumulate additively, module fails when the total amount of damage has exceeded a failure level and the level is previously specified as show in Fig. 2. It is of great importance that module is maintained before failure occurs as preventive maintenance. The cumulative process is mentioned briefly and summarized with reliability measures which are useful to damage models.

\subsection{Model description}

To predict the life-time of the reuse module, one of methods is economical evaluation by reducing the expected maintenance cost rate. Seeding the functions that minimize the expected maintenance cost and $T$ the lifetime can be predicted by the functions. In this damage model the functions are $T^{*}$ and $N^{*}$, and will be discussed in this study. Cumulative damage model has been discussed to analyze the optimal replacement about the unit with periodic inspection, and the optimal damage level is analyzed for minimizing the expected replacement cost with repairing process, and the module failure level is a random distribution ${ }^{(20),(21)}$. In this study, the damage model is also discussed to analyze the optimal maintenance time $T^{*}$ and maintenance shock number $N^{*}$ which 
minimizes the expected maintenance cost. Reuse module can be divided by $T^{*}$ and $N^{*}$ to warrant the quality, in this process the module repairing is not considered and the module failure damage level is previously specified.

Suppose that a module is subjected to shock at a nonhomogeneous Poisson process with an intensity function $\lambda(t)$ and a mean-value function $\beta(t)$ and $\beta(t)=\int_{0}^{t} \lambda(x) d x$. Further, it is assumed that the probability that the damage shock occurs is $p$ and the probability that failure shock occurs is $(1-p)$. it is noted that failure shocks occur at non-homogeneous Poisson process with an intensity function $(1-p) \lambda(t)$, and damage shocks occur with an intensity function and a mean-value function $R(t) \equiv p \beta(t)$. Then, the probability of $j$ shocks during $(0, t]$ is $S_{j}(t)$. Further, an amount $W_{j}$ of damages arises from the $j$-th shock and has an identical probability distribution $G(x)$ with finite mean, independent of the number of shocks. It is assumed that these damages are accumulated to the current damage level. Then, the total damage $A_{j}=\sum_{i=1}^{j} W_{j}$ up to the $j$-th shock has

$$
P_{r}\{A(t) \leq x\}=G_{j}(x)
$$

Let $A(t)$ be the total amount of damage of an item at time $t$, then the distribution of $A(t)$ is

$$
P_{r}\{A(t) \leq x\}=\sum_{j=0}^{\infty} G_{j}(x) S_{j}(t)
$$

Where

$$
S_{j}(t)=\left\{[R(t)]^{j} / j !\right\} e^{-R(t)}(j=0,1,2 \cdots)
$$

and its mean time is

$$
T_{m}=\sum_{j=0}^{\infty} G_{j}(x) \int_{0}^{\infty} S_{j}(t) d t
$$

\subsection{Expected maintenance cost rate}

Consider the cumulative damage model, where a module fails when the total damage has exceeded a failure level $K$ where $K$ is previously specified. As preventive maintenance, the module is maintained at time $T$ or at $N$-th shock, whichever occurs first, for this model, we introduce the following maintenance cost: let $c_{t}$ and $c_{n}$ be fixed costs for the respective maintenance at time $T$ and at $N$-th shock, and $c_{k}$ be a fixed cost for maintenance when the total damage has exceeded a level $K$, where $c_{t}<c_{k}$ and $c_{n}<c_{k}$. Further, let $c_{0}(x)$ be an additional cost which is variable for the amount $x$ of damage. The expected cost $C_{E}$ when an item is maintained at time $T$ and or at $N$-th shock is

$$
\begin{aligned}
C_{E}= & \sum_{j=0}^{N-1} S_{j}(T) \int_{0}^{K}\left[c_{t}+c_{0}(x)\right] d G_{j}(x) \\
& +\int_{0}^{T} S_{N-1}(t) \lambda(t) d t \int_{0}^{K}\left[c_{n}+c_{0}(x)\right] d G_{N}(x)(5)
\end{aligned}
$$

And the expected cost when the total damage has exceeded a level $K$ is

$$
C_{K}=\left[c_{k}+c_{0}(K)\right] \sum_{j=0}^{N-1}\left\{G_{j}(K)-G_{j+1}(K)\right\} \int_{0}^{T} S_{j}(t) \lambda(t) d t
$$

The mean time to maintenance is

$$
T_{M}=\sum_{j=0}^{N-1} G_{j}(K) \int_{0}^{T} \frac{[R(t)]^{j}}{j !} e^{-R(t)} d t
$$

Therefore, the expected maintenance cost rate is

$$
C(T, N)=\left(C_{E}+C_{K}\right) / T_{M}
$$

For seeding the safe use time of the reuse module, in the next the functions that how to minimize the expected maintenance cost rate will be discussed.

\section{Life Prediction of Reuse Module with Optimal Maintenance Time}

One of the methods that minimizing the expected is to discuss the optimal maintenance time of the reuse module. Suppose that the reuse module is maintained at only time $T$. Then expected cost is given by $C(T)=\lim _{N \rightarrow \infty} C(T, N)$. To seek the optimal time $T^{*}$ which minimizes $C(T)$, differentiating $C(T)$ with respect to $T$ and setting it equal zero imply

$$
\begin{aligned}
& \left(c_{k}-c_{t}\right)\left\{\lambda Q_{1}(T) \sum_{j=0}^{\infty} G_{j}(K) \int_{0}^{T} S_{j}(t) d t\right. \\
& \left.-\sum_{j=0}^{\infty} S_{j}(T)\left[1-G_{j}(K)\right]\right\} \\
& +\frac{\lambda(T) \sum_{j=0}^{\infty} S_{j}(T) \int_{0}^{K}\left[G_{j}(x)-G_{j+1}(x)\right] d c_{0}(x)}{\sum_{j=0}^{\infty} S_{j}(T) G_{J}(K)} \\
& \times \sum_{j=0}^{\infty} G_{j}(K) \int_{0}^{T} S_{j}(t) d t=c_{t}
\end{aligned}
$$

Where

$$
Q_{1}(T) \equiv\left\{\sum_{j=0}^{\infty} S_{j}\left[G_{j}(K)-G_{j+1}(K)\right\} / \sum_{j=0}^{\infty} S_{j}(T) G_{j}(K)\right.
$$

It is very difficult to discuss an optimal $T^{*}$ analytically. In particular, we assume that a module is subjected to shock at a Poisson with rate $\lambda$ and $c_{0}(x)$ is proportional to an amount of damages, i.e., $c_{0}(x)=c_{0} x, \lambda(t)=\lambda$. Then, $C(T)=\lim _{N \rightarrow \infty} C(T, N)$ and Eq. (9) are rewritten as, respectively,

$$
\begin{aligned}
C(T)= & \left\{c_{k}-\left(c_{k}-c_{t}\right) \sum_{j=0}^{\infty} S_{j}(T) G_{j}(K)\right. \\
& \left.+c_{0} \sum_{j=0}^{\infty} S_{j}(T) \int_{0}^{K}\left[1-G_{j}(x)\right] d x\right\} \\
& / \sum_{j=0}^{\infty} G_{j}(K) \int_{0}^{T} S_{j}(t) d t
\end{aligned}
$$




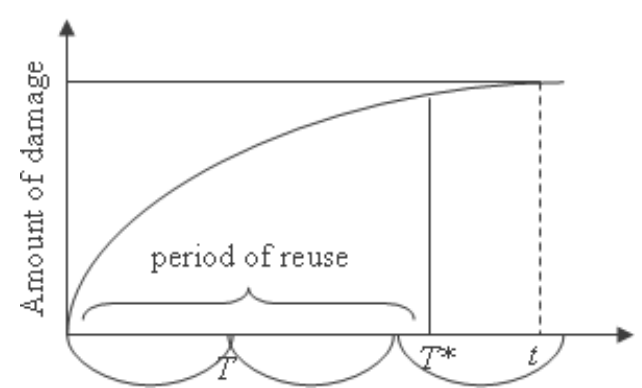

Fig. 3 Period of module reuse about optimal maintenance time

$$
\begin{aligned}
& \left(c_{k}-c_{t}\right)\left\{\lambda Q_{1}(T) \sum_{j=0}^{\infty} G_{j}(K) \int_{0}^{T} S_{j}(t) d t\right. \\
& \left.-\sum_{j=0}^{\infty} S_{j}(T)\left[1-G_{j}(K)\right]\right\} \\
& +c_{0}\left\{\lambda Q_{2}(T) \sum_{j=0}^{\infty} G_{j}(K) \int_{0}^{T} S_{j}(t) d t\right. \\
& \left.-\sum_{j=0}^{\infty} S_{j}(T) \int_{0}^{K}\left[1-G_{j}(X)\right] d x\right\}=c_{1}
\end{aligned}
$$

where

$$
\begin{aligned}
& Q_{2}(T) \equiv \frac{\sum_{j=0}^{\infty} S_{j}(T) \int_{0}^{K}\left[G_{j}(x)-G_{j+1}(x+1)\right] d x}{\sum_{j=0}^{\infty} S_{j}(T) G_{J}(K)} \\
& H_{j}(t) \equiv \frac{(\lambda t)^{j}}{j !} e^{-\lambda t}(j=0,1,2, \cdots)
\end{aligned}
$$

If $c_{0}=0$ then Eq. (12) is

$$
\begin{aligned}
& \left\{\lambda Q_{1}(T) \sum_{j=0}^{\infty} G_{j}(K) \int_{0}^{T} S_{j}(t) d t-\sum_{j=0}^{\infty} S_{j}(T)\left[1-G_{j}(K)\right]\right\} \\
& =c_{t} /\left(c_{k}-c_{t}\right)
\end{aligned}
$$

If $Q_{1}(T)$ is strictly increasing, then the left-hand side in Eq. (14) is also strictly increasing, from 0 to $Q_{1}(\infty)[1+$ $M(K)]-1$, and

$$
Q_{1}(\infty) \equiv \lim _{T \rightarrow \infty} Q_{1}(T), M(K) \equiv \sum_{j=1}^{\infty} G_{j}(K)
$$

which represents the mean number of shocks until the total damage exceeds a failure level $K$. Thus, if

$$
Q_{1}(\infty)[1+M(K)]>c_{t} /\left(c_{k}-c_{t}\right)
$$

there exists a finite and unique $T^{*}$ which satisfies Eq. (14). Therefore the optimal maintenance cost and time can be discussed by the failure level $K$.

As show in Fig. $3 t$ is failure point, at this point the failure level has exceeded the level $K . T^{*}$ is optimal maintenance time, and $T$ is schedule maintenance time. If there are many times about the schedule maintenance time before the amount of the damage exceeds the failure level, the total maintenance cost will turn large. Therefore, the optimal maintenance time $T^{*}$ is calculated by the damage model. If the model is reused between the $t$ and $T^{*}$, the quality is uncertain and fails easily because the amount of damage is near to the failure level. Thus it is considered that the period of the module reuse is between the 0 and $T^{*}$. If the distribution of the amount of damage is known the optimal time $T^{*}$ can be predicted.

\section{Life Prediction of Module Reuse with Optimal Number of Shock}

The second method is to seed the optimal number of shock that minimizing the expected maintenance cost. Through the optimal number of shock the safe use time of the reuse module can be explored. Suppose the expected cost rate when module is maintained at only $N$-th shock is $C(N)=\lim _{T \rightarrow \infty} C(T, N)$ then

$$
\begin{aligned}
C(N)= & \left\{\left[c_{k}+c_{0}(K)\right]\left[1-G_{N}(k)\right]\right. \\
& \left.+\int_{0}^{k}\left[\left[c_{n}+c_{0}(x)\right] d G_{N}(x)\right]\right\} \\
& / \sum_{j=0}^{N-1} G_{j}(K) \int_{0}^{\infty} H_{j}(t) d t
\end{aligned}
$$

Forming the inequality $C(N+1)-C(N) \geq 0$ to seek an optimal number $N^{*}$ which minimizes $C(N)$. Suppose that $c_{0}(x)=c_{0} x, \lambda(t)=\lambda$, then, we have $C(N)$ and the Eq. (19)

$$
\begin{aligned}
& C(N)=\left\{c_{k}-\left(c_{k}-c_{n}\right) G_{N}(k)+c_{0} \int_{0}^{k}\left[1-G_{N}(x)\right] d x\right\} \\
& /(1 / \lambda) \sum_{j=0}^{N-1} G_{j}(K) \\
&\left(c_{K}-c_{n}\right)\left\{\frac{G_{N}(K)-G_{N+1}(K)}{G_{N}(K)} \sum_{j=0}^{N-1} G_{j}(K)-\left[1-G_{N}(K)\right]\right\} \\
&+c_{0}\left\{\frac{\int_{0}^{K}\left[G_{N}(x)-G_{N+1}(x)\right] d x}{G_{N}(K)} \sum_{j=0}^{N-1} G_{j}(K)\right. \\
&\left.-\int_{0}^{K}\left[1-G_{N}(x)\right] d x\right\} \geq c_{n}
\end{aligned}
$$

if $c_{0}=0$ then Eq. (19) is

$$
\frac{G_{N}(K)-G_{N+1}(K)}{G_{N}(K)} \sum_{j=0}^{N-1} G_{j}(K)-\left[1-G_{N}(K)\right] \geq \frac{c_{n}}{c_{k}-c_{n}}
$$

Denote the left-hand side in Eq. (18) by $U(N)$. Then, we have

$$
U(N)-U(N-1)=\left[\frac{G_{N}(K)}{G_{N-1}(K)}-\frac{G_{N+1}(K)}{G_{N}(K)}\right] \sum_{j=0}^{N-1} G_{j}(K)
$$




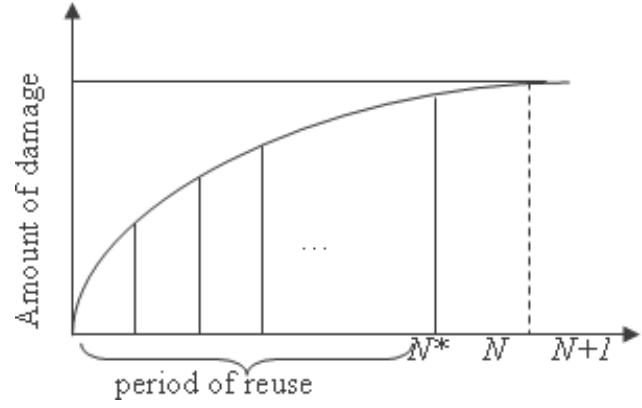

Fig. 4 Period of module reuse about optimal number of shock

Thus, if $G_{j+1} N / G_{j}(N)$ is strictly decreasing in $j$, then $U(N)$ is also strictly increasing, and

$$
\begin{aligned}
& U(\infty)=\lim _{N \rightarrow \infty} U(N)=Q_{3}(\infty)[1+M(K)]-1 \\
& Q_{3}(\infty)=\lim _{N \rightarrow \infty}\left[G_{N}(K)-G_{N+1}(K)\right] / G_{N}(K)
\end{aligned}
$$

therefore, if

$$
Q_{3}(\infty)[1+M(K)] \geq c_{k} /\left(c_{k}-c_{n}\right)
$$

then, there exists an unique minimum $N^{*}$ which satisfies Eq. (18).

As show in Fig. 4 the failure level is between number of shock $N$ and $N+1$. $N^{*}$ is optimal number of damage shock, and at this point the maintenance cost rate is minimal. As the method of optimal time if the module is reused between the $N^{*}$ and the $N$, the module fails easily and it is not economical. The reuse period should be chosen between 0 and $N^{*}$. If the distribution of the amount of damage is known the optimal time $N^{*}$ can be predicted.

\section{Numerical Examples of Optimal Maintenance Methods}

In this section, the two methods are discussed by numerical examples to evaluate period of reuse, and the results are compared in the end of the section.

Computing the optimal method numerically when $c_{0}(x)=c_{0} x, \lambda(t)=\lambda$ and suppose the amount of damage of the module again has an exponential distribution

$$
\begin{aligned}
& G(x)=1-e^{-\mu x} \text { and } G_{j}(k)=\sum_{i=j}^{\infty}\left[(\mu k)^{i} / i !\right] e^{-\mu k} \\
& Q_{1}(T)=1-\left\{\left[\sum_{j=0}^{\infty} S_{j}(T) G_{j+1}(K)\right] /\left[\sum_{j=0}^{\infty} S_{j}(T) G_{j}(K)\right]\right\}
\end{aligned}
$$

In this case Eq. (12) can be rewritten, denote the left-hand side by $L(T)$, then it is evident that

$$
\begin{aligned}
& L^{\prime}(T)=\lambda\left(c_{k}-c_{t}-\frac{c_{0}}{\mu}\right) Q_{1}^{\prime}(T) \sum_{j=0}^{\infty} G_{j}(K) \int_{0}^{T} H_{j}(t) d t \\
& L_{1}(\infty)=\lim _{T \rightarrow \infty} L_{1}(T)=\mu K\left(c_{k}-c_{t}-\frac{c_{0}}{\mu}\right)
\end{aligned}
$$

Table 1 Optimal time $\lambda T^{*}$ and resulting cost $C\left(T^{*}\right) / \lambda$ with $\mu=$ 1

\begin{tabular}{|l|l|l|l|l|l|l|l|l|}
\hline \multirow{2}{*}{$c_{k} / c_{t}$} & \multicolumn{6}{|c|}{ Average failure damage level about $T^{*}(\mu K)$} \\
\cline { 2 - 9 } & \multicolumn{2}{|c|}{150} & \multicolumn{2}{|c|}{300} & \multicolumn{2}{|c|}{500} & \multicolumn{2}{|c|}{700} \\
\cline { 2 - 9 } & $\lambda T^{*}$ & $C\left(T^{*}\right) / \lambda$ & $\lambda T^{*}$ & $C\left(T^{*}\right) / \lambda$ & $\lambda T^{*}$ & $C\left(T^{*}\right) / \lambda$ & $\lambda T^{*}$ & $C\left(T^{*}\right) / \lambda$ \\
\hline 100 & 98.1 & 0.0171 & 221 & 0.0079 & 394 & 0.0045 & 572 & 0.0031 \\
\hline 200 & 95.3 & 0.0173 & 217 & 0.0080 & 389 & 0.0046 & 565 & 0.0032 \\
\hline 500 & 92.0 & 0.0179 & 212 & 0.0081 & 382 & 0.0046 & 558 & 0.0032 \\
\hline 1000 & 89.6 & 0.0180 & 209 & 0.0082 & 377 & 0.0046 & 552 & 0.0032 \\
\hline
\end{tabular}

Note that $G_{j+1} N / G_{j}(N)$ is strictly decreasing in $j$, thus $Q_{1}(T)$ is strictly increasing, and hence, $L_{1}(T)$ is also strictly increasing for $c_{k}-c_{t}-\left(c_{0} / \mu\right)>0$ therefore, if

$$
c_{k}-c_{t}-\left(c_{0} / \mu\right)>c_{t} / \mu K
$$

then there exists a finite and an unique $T$, and the resulting expected cost is

$$
C_{1}\left(T^{*}\right)=\lambda\left[\left(c_{0} / \mu\right)+\left(c_{k}-c_{t}\right) Q_{1}\left(T^{*}\right)\right]
$$

Next, we compute an optimal number $N *$ which minimizes $C(N)$ and Eq. (20) becomes

$$
\begin{aligned}
& \left(c_{k}-c_{n}\right)\left\{\left[1-G_{N+1}(K) / G_{N}(K)\right] \sum_{j=0}^{N-1} G_{j}(K)-\left[1-G_{j}(K)\right]\right\} \\
& +\frac{c_{0}}{\mu}\left\{\left[G_{N+1}(K) / G_{N}(K)\right] \sum_{j=0}^{N-1} G_{j}(K)-\sum_{j=0}^{N} G_{j}(K)\right\} \geq c_{3}
\end{aligned}
$$

Denote the left-hand side by $L_{2}(N)$. Then

$$
\begin{aligned}
& L_{2}(N)-L_{2}(N-1)=\left(c_{k}-c_{n}-\frac{c_{0}}{\mu}\right) \\
& \times\left[\frac{G_{N}(K)}{G_{N-1}(K)}-\frac{G_{N+1}(K)}{G_{N}(K)}\right] \sum_{j=0}^{N-1} G_{j}(K) \\
& L_{2}(\infty)=\lim _{N \rightarrow \infty} L_{2}(N)=\mu K\left[c_{k}-c_{n}-\left(c_{0} / \mu\right)\right]
\end{aligned}
$$

Therefore, if

$$
c_{0}-c_{n}-\left(c_{0} / \mu\right)>c_{n} / \mu K
$$

then there exists an unique minimum $N$, and conversely, if

$$
c_{0}-c_{n}-\left(c_{0} / \mu\right) \leq c_{n} / \mu K
$$

then $N \rightarrow \infty$ since $G_{j+1} N / G_{j}(N)$ is strictly decreasing in $j$. Then two tables are given about maintenance level taking shock rate $\lambda$ into consideration.

Then, according to Eq. (30) Tables 1 is given between failure damage level and maintenance level taking shock rate $\lambda$ into consideration. If parameters $\mu K$ and $c_{k} / c_{t}$ are previously specified, the optimal maintenance level can be calculated. In Table $1 \lambda T^{*}$ is the optimal maintenance level, if the failure damage level $\mu K$ of reuse module is known, then $\lambda T^{*}$ and the maintenance cost can be calculated for every value of $c_{k} / c_{t}$. In the same way, the optimal maintenance level about $N^{*}$ can be calculated as the value 
Table 2 Optimal shock number $N^{*}$ and resulting cost $C\left(N^{*}\right) / \lambda$ with $\mu=1$

\begin{tabular}{|l|l|l|l|l|l|l|l|l|}
\hline \multirow{2}{*}{$c_{k} / c_{n}$} & \multicolumn{6}{|c|}{ Average failure damage level about $N^{*}(\mu K)$} \\
\cline { 2 - 9 } & \multicolumn{2}{|c|}{150} & \multicolumn{2}{|c|}{300} & \multicolumn{2}{|c|}{500} & \multicolumn{2}{c|}{700} \\
\cline { 2 - 9 } & $N^{*}$ & $C\left(T^{*}\right) / \lambda$ & $N^{*}$ & $C\left(T^{*}\right) / \lambda$ & $N^{*}$ & $C\left(T^{*}\right) / \lambda$ & $N^{*}$ & $C\left(T^{*}\right) / \lambda$ \\
\hline 100 & 110 & 0.0160 & 241 & 0.0075 & 421 & 0.0044 & 605 & 0.0031 \\
\hline 200 & 108 & 0.0161 & 238 & 0.0076 & 417 & 0.0044 & 600 & 0.0031 \\
\hline 500 & 105 & 0.0164 & 234 & 0.0076 & 412 & 0.0044 & 594 & 0.0031 \\
\hline 1000 & 103 & 0.0165 & 231 & 0.0077 & 409 & 0.0044 & 590 & 0.0031 \\
\hline
\end{tabular}

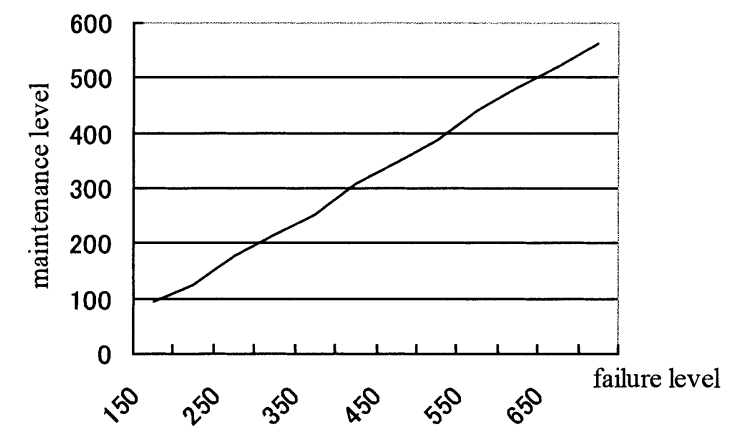

Fig. 5 The relation between maintenance level and module failure level

example in Table 2. As numerical example, the minimal maintenance cost depends on the damage level and has little relation with $c_{k} / c_{t}$ or $c_{k} / c_{n}$. $c_{k}$ is a certain value for a module and it is the upper limit of maintenance cost.

The result helps divide the reuse modules, the operating record is easy for answering the question, if the maintenance damage and the function $\lambda(t)$ of the module can be known. For example, a vehicle module brake pad, whose life-time is connected with the mileage and used-time, in this case if the $\lambda=0.0093$ and the module failure damage level is 700 and maintenance damage level is 572 , then the optimal time is $572 / 0.0093=61505$ hours, 7.02 years and actually the life-time of the brake pad is approximately eight years, and $572 / 700=81.714 \%$, therefore, the module should be maintained when the damage level exceed to $81.714 \%$, and it corresponds to the practical use.

It is obvious when to maintain the module according to the relation between the maintenance damage level and the average failure damage level, and that the maintenance cost is minimal. In module reuse if the amount of cumulative damage level exceeds the maintenance level, the quality can not be guaranteed and causes additional maintenance cost. The maintenance damage level is one of the standards to decide whether disassembled module can be reused or not. Hence, the upper limit quality of remanufactured product affects the user buying decision. Two tables show the relation between amount of damage level of optimal maintenance and average failure damage level, at this point the module should be maintained to guaran- tee the quality. The results of the two quality evaluation methods approach a same tendency. Therefore, the Fig. 5 gives the relation between maintenance level and module failure level.

\section{Conclusions}

Though there are various kinds of the disassembled modules such as household appliance module, machine module, and vehicle module, using the same method to analyze all of the disassembled modules is impossible and every type of module needs a practical possibility inspection method, and the damage model towards the shock type module. Therefore, in this study, a damage model is discussed, and the exponential distribution proved its effectiveness. A reuse module life time analysis method is proposed to minimize the maintenance cost, and the result confirmed verification of the method. It showed a possibility with better reuse potential. In future work, the user buying decision-making will be analyzed considering three elements - quality, price and environmental consideration to promote the remanufactured product consumption.

\section{References}

( 1 ) Steveis, A. and Huuisman, J., An Industry Vision on the Implementation of WEEE and RoHS, International Symposium on Environmentally Conscious Design and Inverse manufacturing, PL-3, (2003).

( 2 ) Takata, S., Umeda, Y., Ogawa, T. and Inamura, T., Framework for Systematic Evaluation of Life Cycle Strategy by Means of Life Cycle Simulation, Proc. of Eco Design 2003: Third Intel. Symposium on Environmentally Conscious Design and Inverse Manufacturing, (2003), pp.198-205.

(3) Kato, S. and Kimura, F., The Product Life Cycle Design Method Using a Strategic Analysis, International Journal of Production Engineering and Computers, Vol.6, No.7 (2004), pp.55-58.

( 4 ) Kimura, F., Kate, S. and Hata, T., Product Modularization for Parts Reuse in inverse Manufacturing, Annals of CIRP, Vol.50, No.1 (2001), pp.89-92.

( 5 ) Kimura, F., Designing Innovative Services and Product Systems Based on the Concept of Total Product Life Cycle Management, Proc. Int. CIRP Design Seminar, KTH, (2001), pp.13-17.

( 6 ) Takata, S. and Kimura, T., Life Cycle Simulation System for Life Cycle Process Planning, Annals of the CIRP, Vol.52, No.1 (2003), pp.37-40.

( 7 ) Ernzer, M., Lindahl, M., Masui, K. and Sakao, T., An International Study on Utilization of Design for Environment Methods (DfE) -A Pre-Study-3th Internal Symposium on Eco Design and Inverse Manufacturing, December 8-11, 1E-1, Tokyo, Japan, (2003).

( 8 ) Nagel, M.H. and Stevels, A.L.N., Design for Environment in the Electronics Industry, Possibilities and Limitations: A Discussion and Evaluation of Product Metrics, Third Internal Symposium on Eco Design and 
Inverse Manufacturing, December 8-11, 1D-, Tokyo, Japan, (2003).

(9) Murayama, T., Tanaka, H. and Oba, F., Real-Time Scheduling in Remanufacturing, Proceedings of 2004 JUSFA, Denver, Colorado, July 19-21, (2004).

(10) Ji, Y., Narita, H., Chen, L.Y. and Fujimoto, H., The Production Scheduling in Inverse Manufacturing, IMCC 2004, September 17-20, Jinan, China, (2004).

(11) Kimura, F., A Methodology for Design and Management of Product Life Cycle Adapted to Product Usage Modes, Proc. 33th CIRP Int. Seminar on Manufacturing Systems, KTH, (2000), pp.139-142.

(12) Seliger, G., Skerlos, S.J., Basdere, B., Zetter, M. and Zettl, M., Design of a Modular Housing Platform to Accommodate the Remanufacturing of Multiple Cellular Telephone Models, Third Internal Symposium on Eco Design and Inverse Manufacturing, December 8 11, 2A-2, Tokyo, Japan, (2003).

(13) Takata, S., Kimura, F., Van Houten, F.J.A.M., Westkaemper, E., Shpitalni, M., Ceglarek, D. and Lee, J., Maintenance: Changing Role in Life Cycle Management, Annals of CIRP, Vol.53, No.2 (2004), pp.634655.

(14) Fujimoto, J., Umeda, Y., Tamura, T., Tomiyama, T. and Kimura, F., Development of Service-Oriented Products Based on the Inverse Manufacturing Concept, Environmental Science and Technology, Vol.37, No.23 (2003), pp.5398-5406, ACS Publications.

(15) Otto, H., Mueller, K. and Kimura, F., Efficient Information Visualization in LCA-Approach and
Examples-, The International Journal of Life Cycle Assessment, Vol.8, No.5 (2003), pp.259-265.

(16) Yim, H. and Herrmann, C., Consumer Behavior on Ecoproduct Why Consumers Do Not Buy an Ecoproduct, Third Internal Symposium on Eco Design and Inverse Manufacturing, December, 3c-6, Tokyo, Japan, (2003).

(17) Pedersen, C.S., Environmental, Health, Safety and Social Life Cycle Management of Moulded-Fibre Packaging for Consumer Electronics, Third Internal Symposium on Eco Design and Inverse Manufacturing, December, 3c-7, Tokyo, Japan, (2003).

(18) Matsuda, M. and Kimura, F., Tolerance Modeling Using the Feature Based Product Model for Prediction of Used Parts Deterioration, Feature Based Product Life Cycle Modeling, (2002), pp.147-164, Kluwer Academic Publisher.

(19) Suzuki, H., Matsuda, M. and Kimura, F., Prediction of Used Parts Deterioration Based on a Tolerance Model, CIRP Journal of Manufacturing Systems, Vol.31, No.3 (2002), pp.175-181.

(20) Satow, T., Yasui, K. and Nakagawa, T., Optimal Garbage Collection Policies for a Database in a Computer System, RAIRO-Operations Research, Vol.30 (1996), pp.359-372.

(21) Sheu, S.H., Kuo C.M. and Nakagawa, T., Extended Optimal Age Replacement Policy with Minimal Repair, RAIRO-Operations Research, Vol.27 (1993), pp.337351. 